\title{
Effect of post-annealing on the plasma etching of graphene-coated-copper $\dagger$
}

\author{
L. S. Hui, ${ }^{a}$ E. Whiteway, ${ }^{b}$ M. Hilke ${ }^{b}$ and A. Turak ${ }^{\star a}$
}

Received 24th May 2014, Accepted 13th June 2014

DOI: $10.1039 / c 4 f d 00118 d$

High temperature deposition of graphene on $\mathrm{Cu}$ by chemical vapor deposition can be used to produce high quality films. However, these films tend to have a non-equilibrium structure, with relatively low graphene adhesion. In this study, samples of graphene grown on copper foils by high temperature CVD were post-deposition annealed at temperatures well below the critical temperature of $\mathrm{Cu}$. Resistance to etching under plasma was examined to assess the mechanical robustness of the graphene on the $\mathrm{Cu}$ surface, analyzed using optical and Raman microscopies. We found a correlation between the post-annealing time and etching time for the complete removal of graphene from $\mathrm{Cu}$. Etching rates, minimum etch times, and surface appearance were observed to vary depending on the etching plasma (air, oxygen or nitrogen). Oxygen plasmas were found to be the least aggressive, emphasizing the improved adhesion with post-annealing treatments. Our results imply that the etching of graphene on $\mathrm{Cu}$, and hence the adhesion of graphene, can be controlled by proper annealing and choice of plasma gas.

\section{Introduction}

Due to its chemical inertness, excellent mechanical strength, and high electrical conductivity graphene has been a widely studied material since its discovery in 2004. ${ }^{1-3}$ Potential applications include its use as electrodes, ${ }^{4-6}$ charge carrier transport layers, ${ }^{7-9}$ lubricants, ${ }^{10}$ impermeable barriers, ${ }^{11,12}$ and in nanoelectromechanical systems (NEMS). ${ }^{13}$

Though much research has focused on understanding the electronic and thermal properties of graphene, ${ }^{1-3}$ the mechanical properties have been less widely explored. However, recent work suggests that the mechanical and friction properties of graphene are just as impressive as the electronic properties. ${ }^{\mathbf{1 4}, \mathbf{1 5}}$ Despite recent excellent work, there still exists some controversy on the strength

\footnotetext{
${ }^{a}$ Department of Engineering Physics, McMaster University, Hamilton, Ontario, L8S 4L7, Canada. E-mail: turaka@mcmaster.ca

${ }^{b}$ Department of Physics, McGill University, Montréal, H3A 2T8, Canada

$\dagger$ Electronic supplementary information (ESI) available. See DOI: 10.1039/c4fd00118d
} 
of adhesion of chemical vapor deposited (CVD) graphene, especially on $\mathrm{Cu}$ surfaces. ${ }^{16-18}$

CVD is an excellent low cost method of producing large area graphene with controlled thickness and high quality. ${ }^{\mathbf{1 9 , 2 0}}$ Though the film quality is high, CVD graphene grown on $\mathrm{Cu}$ foil at high deposition temperatures $\left(>1000{ }^{\circ} \mathrm{C}\right)$ tends to have a non-equilibrium structure, ${ }^{21}$ characterized by wrinkles and folds. ${ }^{16,21-23}$ Deposition at high temperatures leads to rapid contraction of graphene upon cooling $^{21,23,24}$ due to the mismatch in the thermal expansion coefficients between $\mathrm{Cu}$ and graphene. ${ }^{25,26}$ The Chen group ${ }^{27,28}$ has recently used high temperature post-deposition annealing to recover the equilibrium structure of graphene on $\mathrm{Cu}^{22}$ This equilibrium structure has been found to have much higher adhesion energies ${ }^{17}$ than those found on wrinkled samples. ${ }^{16}$

This study examined the impact of post-deposition annealing (post-annealing) on the graphene-Cu system when exposed to etching plasmas. Post-deposition annealing, even at modest temperatures $\left(<200{ }^{\circ} \mathrm{C}\right)$, appears to be key in manipulating the adhesion strength of graphene on $\mathrm{Cu}$, reflected by the increased etching times required to completely remove CVD graphene from the $\mathrm{Cu}$ surface. Plasma etching, therefore, may be an easy and effective method of examining the impact of post-deposition treatments on graphene systems.

\section{Experimental}

Graphene was synthesized by chemical vapour deposition (CVD) on commercially available $25 \mu \mathrm{m}$ copper foils (Alfa Aesar). The foils were first chemically treated with acetic acid and annealed for 4 hours at $1078{ }^{\circ} \mathrm{C}$ under a flow of $8 \mathrm{sccm}$ hydrogen gas in order to prepare the copper surface. The temperature was maintained at $1078{ }^{\circ} \mathrm{C}$ during a 4 min CVD growth phase where a gas flow of 1.2 sccm methane was introduced. A long annealing time and short growth period were chosen in order to limit the size and density of the graphene domains. To observe the graphene flakes, oxidation of the $\mathrm{Cu}$ surface is necessary to provide contrast between the graphene coated and uncoated portions of the $\mathrm{Cu}$ surface. Samples were annealed in batches on a hot plate (Pace Heat Wave) in air at $180{ }^{\circ} \mathrm{C}$ with different annealing times of $t=2,4,9,15,30$, and $60 \mathrm{~min}$. The groups of samples with different annealing times were then separated into batches for air (unfiltered laboratory air), nitrogen (Alphagaz, 99.999\%) and oxygen (Alphagaz, 99.999\%) plasma etching. The plasma etching was performed in a Harrick Plasma PDC-001 plasma cleaner system and a Thierry Plasma Zepto Low Pressure System with a $13.56 \mathrm{MHz}$ R.F. generator at $29.6 \mathrm{~W}$. The plasma chamber was held at a base pressure of $70 \mathrm{mTorr}$ prior to target gas filling. During etching, a gas flow rate of $30 \mathrm{sccm}$ was maintained. The samples were progressively etched from 2-260 min with time intervals of a minimum of $1 \mathrm{~min}$ and were observed under an optical microscope (Leica DMR microscope) to detect the first disappearance of contrast. For the case of $\mathrm{N}_{2}$ plasma etching, a second post-annealing step of the etched samples was needed to show the contrast of $\mathrm{Cu}$-graphene after treatment. The time intervals from the etching were accumulated to determine the minimum time required to completely remove the graphene from the $\mathrm{Cu}$ surface, designated as the critical etching time. Raman spectra were collected using a Renishaw inVia spectrometer at $514 \mathrm{~nm}$ laser excitation, with a $20 \times$ objective and $1200 \mathrm{l}$ per $\mathrm{mm}$ grating. The laser power was $20 \mathrm{~mW}$ to prevent damage to the graphene. All of the 
spectra were background corrected to remove the $\mathrm{Cu}$ photoluminescence excitation at $514 \mathrm{~nm}$ (ref. 29) (see the ESI†).

\section{Results and discussion}

Though exposure to plasma is often used to modify the electronic properties of graphene layers, ${ }^{30-34}$ under sufficiently high doses bombardment with energetic particles can also completely etch away the graphene layer. The etching time for the complete removal of any residue is generally correlated to the strength of the adhesion of the coating on the surface. ${ }^{35}$ Therefore, plasma interactions are a potential method for probing the mechanical strength of graphene-copper interfaces. As our interest concerns the robustness of the graphene against plasma etching, partially covered substrates were desired. The CVD process was tuned to produce square graphene flakes on the $\mathrm{Cu}$ foil with $\sim 30-100 \mu \mathrm{m}$ widths.

\section{Impact of etching times}

Fig. 1 shows the background subtracted Raman spectra for the as-grown graphene, and the resultant spectra after etching in $\mathrm{O}_{2}$ for various times. The pristine sample shows both the characteristic features of graphene, the $\mathrm{G}$ and 2D peaks at $1587 \mathrm{~cm}^{-1}$ and $2705 \mathrm{~cm}^{-1}$ respectively. The G peak corresponds to the first order degenerate phonon energy, $E_{2 \mathrm{~g}}$ mode at the $\Gamma$ point of graphene; ${ }^{36}$ the sharp $2 \mathrm{D}$ peak is a result of the second order phonon intervalley scattering, from the $\pi-\pi^{*}$ transition. ${ }^{36-38}$ The absence of a peak between $\sim 1280$ and $1360 \mathrm{~cm}^{-1}$ suggests that the graphene islands are mostly defect free. ${ }^{36,39}$ The $I_{\mathrm{G}} / I_{2 \mathrm{D} \text { (peak height) }}$ ratio of 0.4 indicates that the graphene flakes on the $\mathrm{Cu}$ surface are mostly few or even single layered. ${ }^{36,40}$ The 2D peak can be fit by a single Lorentzian function (not shown), further supporting the idea that the CVD grown graphene films are mostly single layer, defect free patches on the Cu surface. ${ }^{36}$ Some islands were also observed

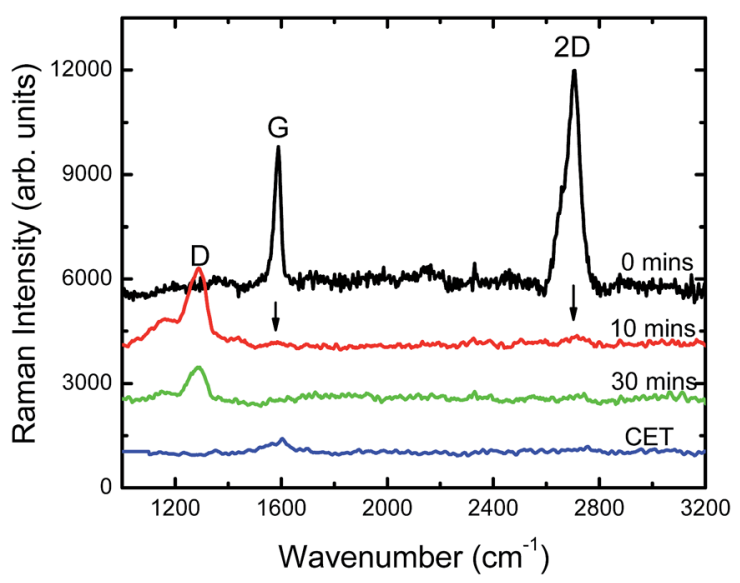

Fig. 1 Raman spectra for CVD graphene on Cu foil at etching times of 0, 10, 20 and 201 (CET) min, showing the evolution of the etching process for $\mathrm{O}_{2}$ plasmas. The critical etching time is defined as the complete removal of graphene, as determined by optical methods. 
with slightly higher $I_{\mathrm{G}} / I_{2 \mathrm{D}}$ ratios and a shoulder on the $2 \mathrm{D}$ peak, suggesting that some flakes are likely multilayered. ${ }^{36}$

After a short etch in $\mathrm{O}_{2}$ plasma, significant $\mathrm{sp}^{3}$ defects were introduced with a strong $\mathrm{D}$ band appearing at $1290 \mathrm{~cm}^{-1}$. $^{\mathbf{3 6 , 4 1}}$ Though much reduced, both the $\mathrm{G}$ and $2 \mathrm{D}$ bands are still barely visible as shown by the arrows in Fig. 1 . There may also be a merging of the $\mathrm{G}$ and $\mathrm{D}^{\prime}$ broad bands at $1590 \mathrm{~cm}^{-1}$, which is attributed to the $\mathrm{C}-\mathrm{H} \mathrm{sp}{ }^{3}$ hybridization defect and the overtone of the D mode activated after plasma treatment. ${ }^{22,27}$ These changes suggest that the plasma initially introduces defects into the graphene rather than removing it from the $\mathrm{Cu}$, as is often seen in lower dose plasma processing used to tune the electronic configuration of graphene flakes. ${ }^{30}$ Another explanation for the appearance of the strong $\mathrm{D}$ band is the formation of graphene oxide, though that is generally accompanied by an equally large G peak, which was not observed here. ${ }^{42}$ Further etching eliminates the G, $\mathrm{D}^{\prime}$ and $2 \mathrm{D}$ signals and reduces the scattering intensity of the $\mathrm{D}$ band, indicating that graphene is being removed from the surface. Finally, after the critical etching time (CET), there is no more evidence of graphene in the Raman spectrum. This fully etched condition of the graphene was confirmed by optical microscopy.

As received, the graphene on $\mathrm{Cu}$ foil is without any contrast in the forward scattering condition. $\mathrm{Cu}$, however, is prone to oxidation in air even at low temperatures. Graphene has been widely reported as an excellent passivation layer, ${ }^{11,12}$ protecting the surface from chemical oxidation as the densely packed benzene-ring structures make graphene impermeable to most gases, including helium. ${ }^{43,44}$ Mild oxidation, from short post-annealing treatments in air or even exposure to oxidizing plasmas, can therefore be used to visualize the graphene islands (see Fig. 2(a)). The contrast results from the different light scattering intensities and refractive indices of cuprous oxide and the unoxidized $\mathrm{Cu}$ protected by the graphene. The oxidation contrast, therefore, can be used as a method of tracking the progress of the etching. Fig. 2 shows the effects of the

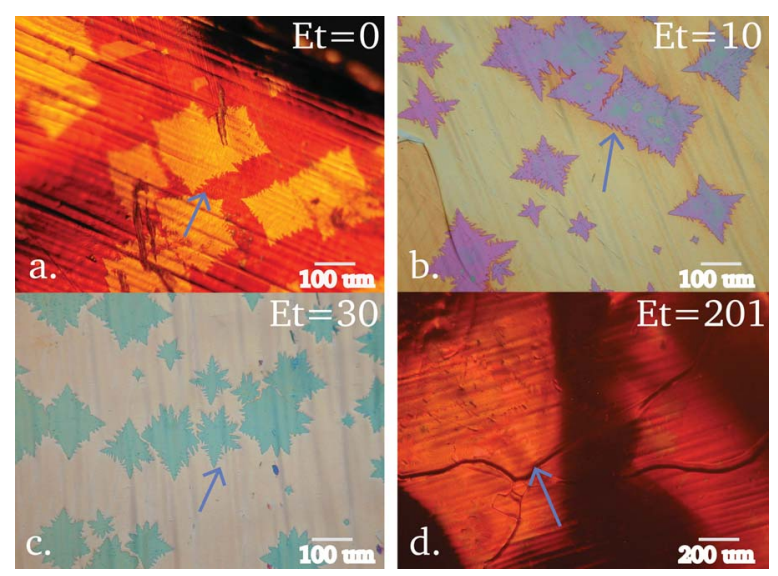

Fig. 2 Optical micrographs of CVD graphene on Cu foils. (a) As-grown sample, annealed to show the contrast between bare and graphene covered regions. (b-d) The same sample after etching in $\mathrm{O}_{2}$ plasma for 10, 30 and 201 min. Etching time is denoted as "Et" in the figures. The blue arrows indicate the contrast showing the graphene islands. The final panel was used to determine the critical etching time (CET). 
etching procedure on the graphene islands, supporting the findings suggested by the Raman data. After short etching times, the graphene is still visible, and then becomes progressively less visible as the etching progresses. Finally, the critical etching time (CET) is determined as the point where almost no contrast is visible after etching, suggesting complete removal of the graphene layer (Fig. 2(d)).

After the CET, there is still a Raman feature visible at the $\mathrm{G} / \mathrm{D}^{\prime}$ location of $\sim 1580 \mathrm{~cm}^{-1}$ (Fig. 1). However, the eigenvector of the $\mathrm{G}$ mode involves the in-plane bond-stretching motion of pairs of $\mathrm{sp}^{2} \mathrm{C}$ atoms, and does not require the presence of six-fold rings. ${ }^{45}$ Without the accompanying $2 \mathrm{D}$ peak, which needs six-fold rings to be excited, ${ }^{45}$ this cannot be taken as a feature of graphene, and is most likely due to $\mathrm{C}=\mathrm{C}$ residues on the surface. As can be seen in Fig. 3, after the CET as determined from optical observations, Raman measurements show no indication of graphene on the surface for $\mathrm{N}_{2}, \mathrm{O}_{2}$ and unfiltered laboratory air etching plasmas. This suggests that the optical method is adequate to determine the CET for complete removal of graphene from the $\mathrm{Cu}$ surface, and the CET can be considered to be roughly proportional to the strength of the interaction between graphene and the $\mathrm{Cu}$ surface. Optical determination of the CET is straightforward for $\mathrm{O}_{2}$ and air plasmas, as exposure to the plasma also oxidized the $\mathrm{Cu}$ surface; however, identification of the CET for $\mathrm{N}_{2}$ plasma required a post-etching anneal of the etched samples to show the contrast of the $\mathrm{Cu}$-graphene after treatment. It is likely therefore that the visual method leads to an over estimation of the real CET for $\mathrm{N}_{2}$ plasmas. This was confirmed with Raman measurements where graphene patches were visible optically, but there was no graphene signature (see next sections).

\section{Effect of post-deposition annealing}

CVD graphene grown on $\mathrm{Cu}$ foil at high deposition temperatures, such as those in this study, tends to have a non-equilibrium structure. ${ }^{21}$ Egberts et al. ${ }^{16}$ recently showed that the work of adhesion for graphene on $\mathrm{Cu}$ is low for such systems. The Chen group ${ }^{27,28}$ have been able to use high temperature post-deposition annealing

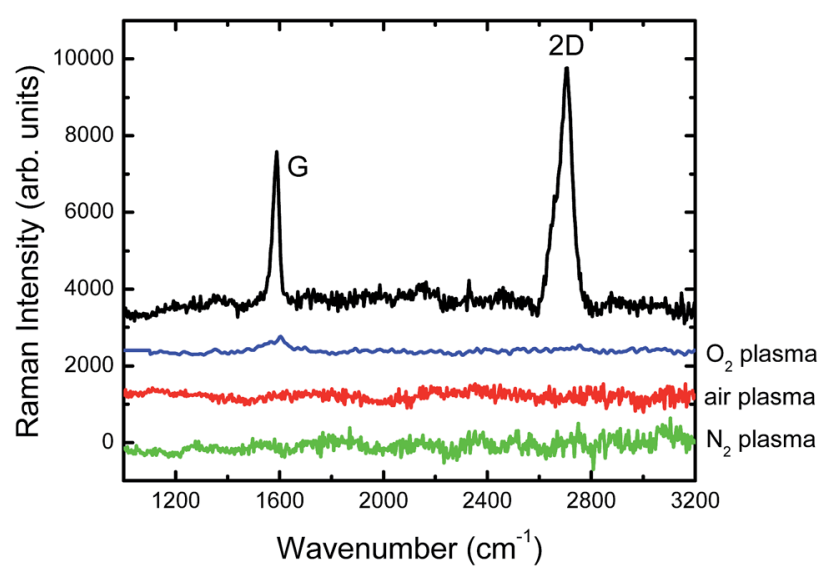

Fig. 3 Raman spectra of as-grown CVD graphene on $\mathrm{Cu}$ and after etching with different gases. 
to recover the crystallinity of the $\mathrm{Cu}$ surface, approaching the equilibrium structure of graphene on $\mathrm{Cu} .{ }^{22}$ High quality equilibrium films have been reported to have very high adhesion strengths. ${ }^{17}$ This suggests that post-deposition annealing might increase the interaction strength between graphene and $\mathrm{Cu}$.

To examine this effect, we performed low temperature $\left(180^{\circ} \mathrm{C}\right)$ post-deposition annealing on our graphene-Cu samples for various annealing times. As described above, short annealing times can make the graphene islands visible. Under further annealing (Fig. 4(b)) the contrast increases, likely due to the enhanced oxidation of unprotected $\mathrm{Cu}$ in the presence of graphene, recently described by

Annealed for 2 mins

Annealed for 30 mins

Annealed for 60 mins

Non-etched

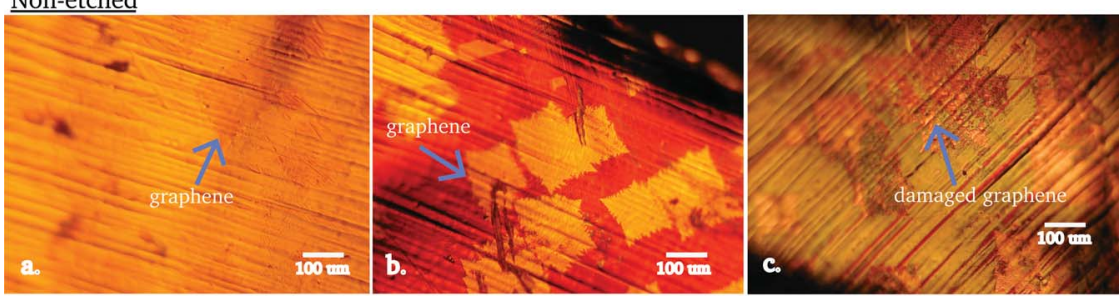

Air plasma treated

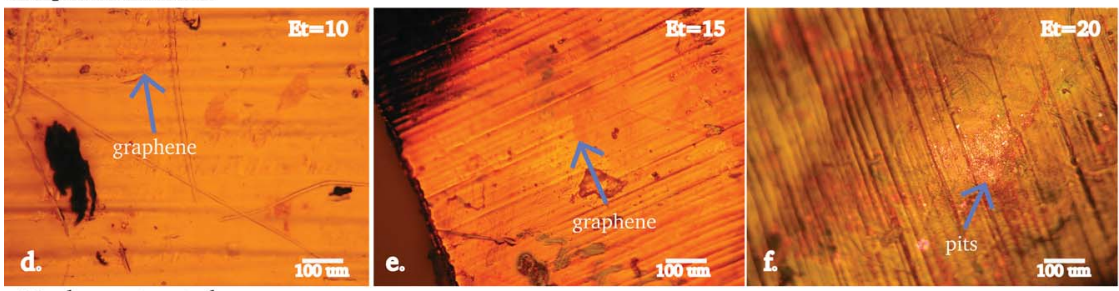

N2 plasma treated
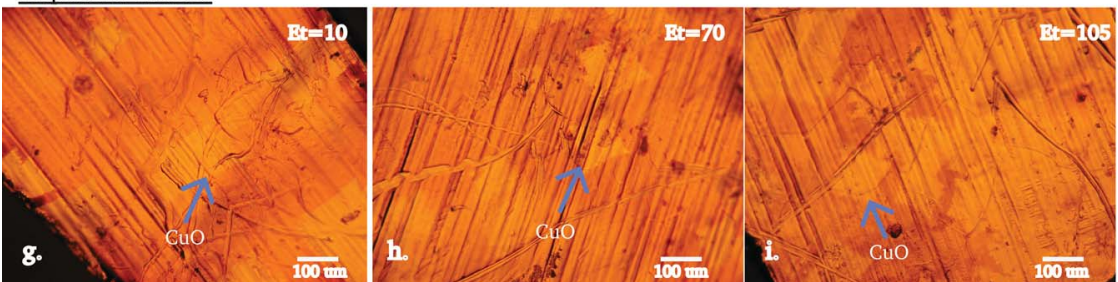

O2 plasma treated
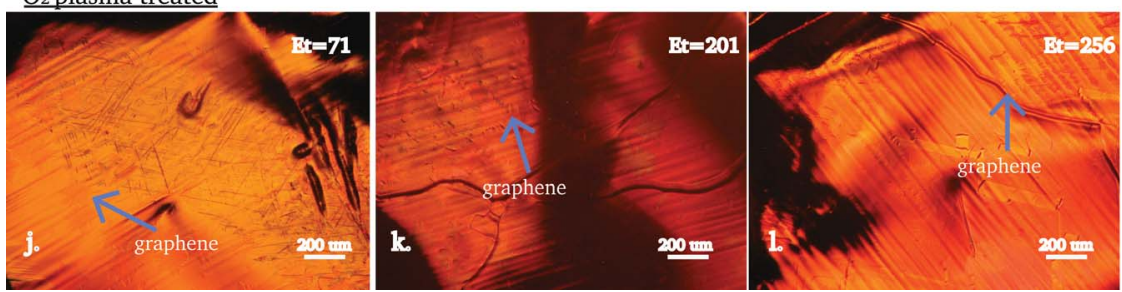

Fig. 4 (a-l) Optical micrographs of CVD graphene on $\mathrm{Cu}$ foil annealed and etched for various times. ( $a, d, g$ and j) Samples post-annealed for $2 \mathrm{~min}$; (b, e, h and k) for 30 min and (c, f, i and l) for $60 \mathrm{~min}$. (a-c) The samples have not been plasma treated; $(d-f)$ treated with air plasma, (g-i) $\mathrm{N}_{2}$ plasma treated and $(j-l) \mathrm{O}_{2}$ plasma treated with the etching time denoted as "et" in the figures. The blue arrows indicate the contrast showing the graphene islands. 
Zhou et al. ${ }^{46}$ Eventually, after annealing for $60 \mathrm{~min}$, in some areas of the sample the graphene island edges are no longer clear, and the islands appear damaged. This is likely to be a result of graphene exfoliation from the surface, due to a mismatch in the thermal expansion coefficients of graphene $\left((-8.0 \pm 0.7) \times 10^{-6}\right.$ $\left.\mathrm{K}^{-1}\right)$ and the $\mathrm{Cu}$ crystals $\left(14 \times 10^{-6} \mathrm{~K}^{-1}\right) \cdot{ }^{25,26}$ With longer annealing times, more material is allowed to expand and the rigid graphene structure is not fully bonded to the surface, exposing some of the $\mathrm{Cu}$ underneath the surface, resulting in a more "messy" oxidation of the $\mathrm{Cu}$ foil. ${ }^{47}$

Fig. 5 shows the Raman spectra for the post-deposition annealed films. In all cases the $\mathrm{G}$ and $2 \mathrm{D}$ bands are both visible, without any $\mathrm{D}$ band development. This suggests that there was neither significant defect nor graphene oxide formation. ${ }^{42,48}$ Post-deposition annealing in fact seems to have very little impact on the graphene islands, even when optically significant damage appears to have occurred. The only notable change is a slight blue shift of the $2 \mathrm{D}$ band observed to occur systematically with annealing time. Though this is characteristic of multilayered graphene, ${ }^{49}$ no peak broadening or change in the $I_{\mathrm{G}} / I_{2 \mathrm{D}}$ ratio (which varies between 0.36 and 0.46 depending on the graphene flakes) were observed. The observed features can be well fitted with a single Lorentzian peak shape with a similar full width at half maximum (FWHM), pointing to defect free, monolayer graphene in all cases. Another possible explanation for the observed shift is the change of strain at the interface. Wang et al. ${ }^{50}$ saw shifts in the $2 \mathrm{D}$ band resulting from the lattice mismatch between SiC and the carbon layer/buffer layer, which has a graphene-like honeycomb lattice that is covalently bonded to the SiC substrate. ${ }^{50} \mathrm{Yu}$ et al. ${ }^{37}$ also observed strain induced shifts in the $2 \mathrm{D}$ band during temperature tuning of growth that they attributed to strain buildup at the interface. A change in the mechanical interaction between the graphene and Cu during post-annealing appears to relieve strain at the interface.

Upon exposure to plasma however, significant differences were observed with differing post-annealing times. Optically, as can be seen in Fig. 4, systematically longer etching times were necessary to fully remove the graphene from the

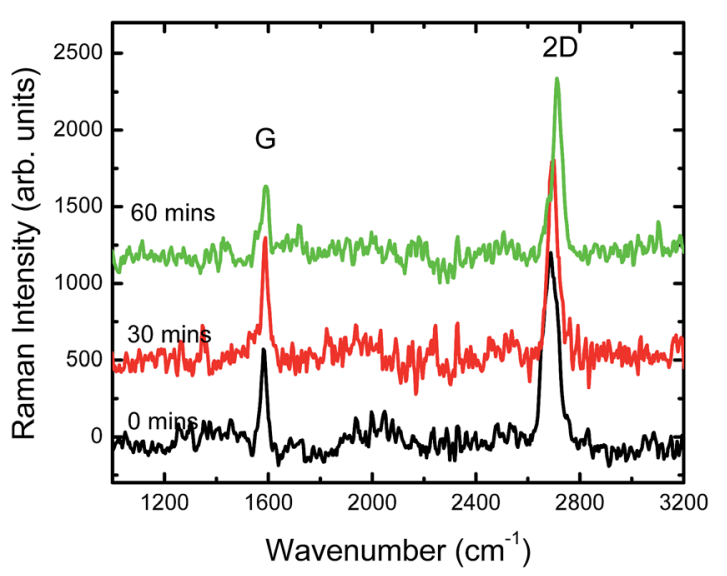

Fig. 5 The Raman spectra of CVD graphene on Cu samples with different post-annealing times at $180^{\circ} \mathrm{C}(0,30$ and $60 \mathrm{~min})$. The spectra were normalized to the $2 \mathrm{D}$ peak intensity at $\sim 2700 \mathrm{~cm}^{-1}$ for comparison. 
substrate with post-annealing, for all of the etching gases. The observed CET values for all the process gases are summarized in Fig. 6 . In all cases, an increased annealing time protects the system against etching. The visually different etching times as a function of annealing, as shown in Fig. 4(d)-(l) support the premise that annealing can be used to control the removal of graphene, by making the structure more robust. It takes much longer in all cases to see the complete removal of graphene from the surface. Fig. 7 shows the Raman spectra for the optically determined CET for $\mathrm{O}_{2}$ plasma with various post-annealing times. Though there is no evidence of graphene or even $\mathrm{C}$ residue on the as-grown samples, even short post-deposition annealing changed the resulting spectra. With only 2 min of annealing, the defect or graphene oxide D band observed previously with 10 min plasma exposure was still visible after 75 min plasma exposure (see the ESI $\dagger$ for non-normalized CET values). Further annealing showed an increasing signal from the $\mathrm{G} / \mathrm{D}^{\prime}$ band, which may be due to $\mathrm{C}=\mathrm{C}$ residues that are not removed even with extremely long etch times (250 min etching for $60 \mathrm{~min}$ post-annealing time). The extremely long etching times and the inability of the plasma to remove carbon residues indicates a strong interaction between the $\mathrm{Cu}$ and the graphene.

\section{Effect of plasma gas}

Though Fig. 6 shows that the trend of increasing CET with annealing holds for all of the process gases, there are significant differences in the measured CET values for the different etch gases. These effects are visible even with normalization to remove sample variation. Using the CET estimations, air is the most aggressive etchant, with $\mathrm{O}_{2}$ and $\mathrm{N}_{2}$ plasmas having decreasing aggressiveness. The effect of post-annealing in protecting the graphene from etch removal is more enhanced the less aggressive the etchant. However the optical measurements are slightly misleading. Raman measurements for the same etching time, shown in Fig. 8,

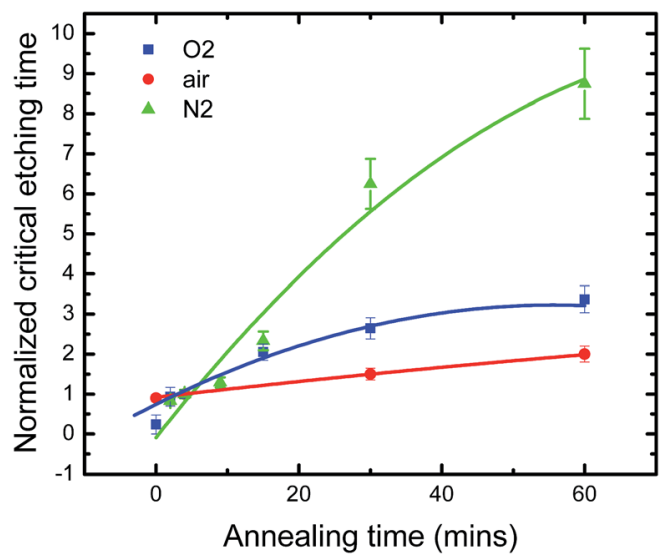

Fig. 6 Normalized CET values for graphene with different post-annealing treatment for various plasmas. The lines are the least squares fit on the data. The CET values have been normalized using the value of annealing time at $4 \mathrm{~min}$, where contrast is visible, to eliminate possible sample discrepancies and emphasize the common trend for all of the process gases. 


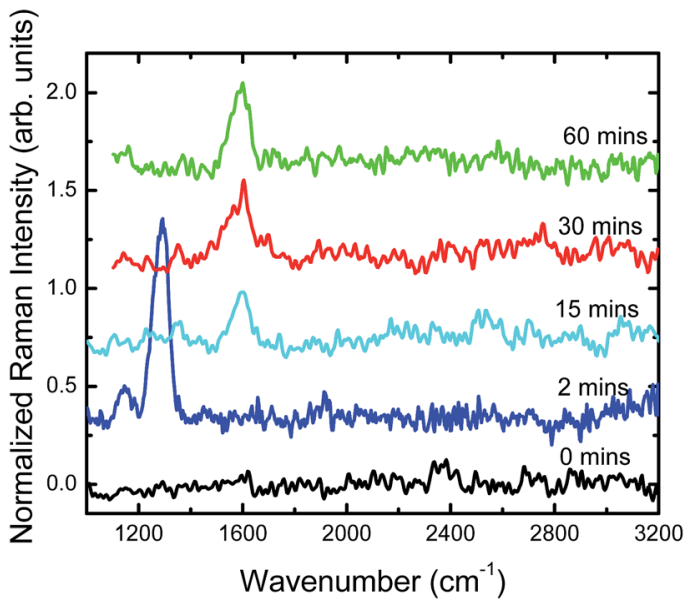

Fig. 7 Raman spectra for CET on CVD graphene on $\mathrm{Cu}$ foils with $\mathrm{O}_{2}$ plasma for various post-annealing times $(0-60 \mathrm{~min})$ at $180^{\circ} \mathrm{C}$.

support the order of the $\mathrm{O}_{2}$ and air plasmas, but completely contradict the behavior with $\mathrm{N}_{2}$ etching.

Fig. 8 confirms that the different process gases have different impacts on the graphene for the same etching time. In this case, $\mathrm{N}_{2}$ plasmas appear to be the most aggressive. No Raman signal is visible from graphene nor are there any $\mathrm{C}-\mathrm{O}$ or $\mathrm{C}=\mathrm{O}$ bands after 10 min etching, suggesting a total destruction of graphene and carbon residues. $\mathrm{N}_{2}$ plasma etching is a physical sputtering process, that has previously been used for nitrogen doping of graphene ${ }^{51}$ and is thought to lead to unsaturated dangling carbon bonds at the edges of the graphene. ${ }^{51}$ The dangling carbon bonds can react with oxygen atoms once exposed to air. However, in this study, no evidence of graphene or graphene oxide was observed, only complete sputter removal of the graphene.

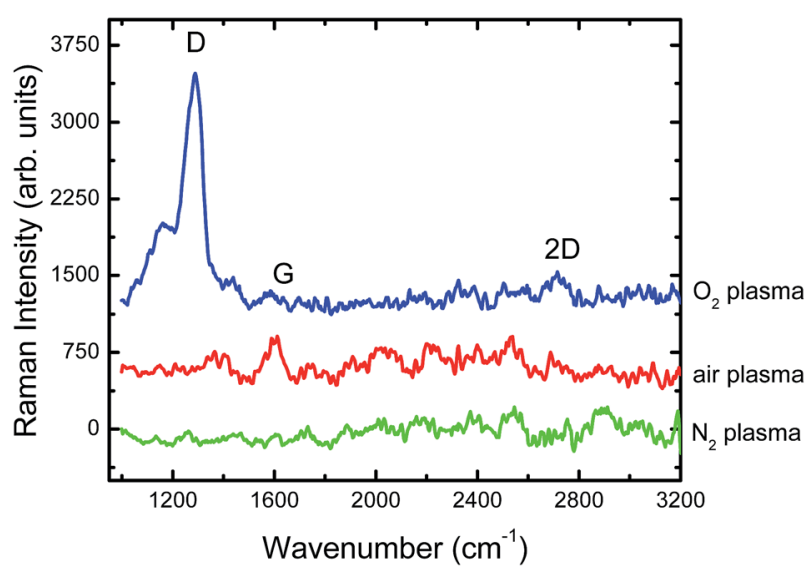

Fig. 8 Raman spectra for CVD graphene on Cu foils with the same post-annealing (30 $\mathrm{min}$ ) and etching times (10 min) with different gases. 
It appears that, while the optical detection for determining the CET works well for air and oxygen plasmas, values of the CET as determined optically are highly overestimated for the $\mathrm{N}_{2}$ plasma. Optically Fig. 4(h) shows that the contrast initially assigned to graphene is still visible for a sample that has been etched for 75 min, under the same annealing conditions as the 10 min etching in Fig. 8. As nitrogen plasma cannot oxidize $\mathrm{Cu}$ during the etching, this contrast is more likely to result from the varying degrees of $\mathrm{Cu}$ oxidation rather than a signature of graphene on the surface. The first annealing step oxidizes the regions without graphene. After interaction with the plasma the graphene is removed, leaving behind regions with different levels of oxidation. During low temperature oxidation, metastable $\mathrm{Cu}_{3} \mathrm{O}_{2}$ forms first followed by $\mathrm{CuO}$, the thermodynamically stable phase of cuprous oxide..$^{52}$ Possibly the regions covered with graphene form the metastable oxide during the second annealing step (i.e. after plasma etching to expose the graphene), while the previously oxidized regions continue to oxidize further. Eventually appearing to be "fully-etched", without any contrast, actually indicates a uniform oxidation or the complete removal of the initially formed oxide by the vigorous physical sputtering action of the plasma. The trend in the observed CET values for $\mathrm{N}_{2}$ plasma may suggest that it takes longer for the cuprous oxide to be sputtered away at longer annealing times.

The Raman data indicate that air plasma is also very aggressive, leaving only the $\mathrm{C}=\mathrm{C}$ residue/G band at $1590 \mathrm{~cm}^{-1}$. Air plasma etching is a very vigorous process, with the CET reached quickly with visible pitting observed on the $\mathrm{Cu}-$ graphene surface (see Fig. 4(f)). This pitting is likely to be due to the effect of hydrogen plasma in air, as Diankov et $a .^{53}$ observed the high selectivity of hydrogen gas on graphene. Pits are not present on the samples etched by $\mathrm{N}_{2}$ (Fig. 4(i)) or $\mathrm{O}_{2}$ plasmas (Fig. 4(l)) for the same annealing time and even for much longer etching times. It is likely that there is true etching occurring with the air plasma, due to the highly reactive $\mathrm{O}^{-}$and $\mathrm{H}^{-}$species, as well as the sputtering observed with nitrogen.

$\mathrm{O}_{2}$ plasmas were the least aggressive, showing the defect structure, D band, and with some $\mathrm{G}$ and $2 \mathrm{D}$ peaks barely visible. As described previously, there could also be a broad $\mathrm{D}^{\prime}$ band, merging with the $\mathrm{G}$ band. The presence of both $\mathrm{D}^{\prime}$ and $\mathrm{D}$ peaks suggests that significant doping and defects are introduced, but likely with little removal of graphene. The gas with the slowest etching rate is thus oxygen, where only chemical etching takes place with $\mathrm{O}^{-}$radical species.

The rates observed for the various plasmas can be attributed to the mechanisms of plasma etching for the different gases. Different plasma gases can have varied interaction mechanisms and diverse ions present in the plasma. ${ }^{53-55}$ Plasma is ignited by the large voltage applied across the electrodes outside the plasma chamber, which causes the free electrons to accelerate rapidly towards the anode. When the electrons collide inelastically with gas molecules, an electron will be removed from the molecules and thus the gas will then become dissociated or ionized. The removed electron continues to be accelerated to the anode and collides with other gas molecules, resulting in a cascading effect. The gas discharge causes excitation and relaxation of gas molecules that release energy in the form of photons, thus causing the glow inside the chamber.

The process of etching is generally considered to be the result of radicals actively breaking chemical bonds and reacting with the substrate surface.$^{56}$ Ions, however, can also cause energetic bombardment or ejection of atoms from the 
surface, a process referred to as sputtering. Oxygen will form highly reactive radicals by molecule dissociation, resulting in etching of the graphene, while nitrogen can be ionized and form ions, ${ }^{57-59}$ thus removing graphene primarily through sputtering. Under exposure to air plasma, graphene is expected to be both etched and sputtered by oxygen, hydrogen, nitrogen, carbon and other atoms of trace gases present in air..$^{55}$ As there is high a percentage of nitrogen in air (78\%), one might expect the air plasma etching process to be dominated by nitrogen. However, pure $\mathrm{N}_{2}$ plasma showed a much faster etching compared to air and oxygen plasmas.

\section{Possible mechanisms for increased adhesion strength with annealing}

The time required for the complete removal of any residue by etching can be broadly correlated to the strength of the adhesion of the coating on the surface. ${ }^{35}$ The observed increase in CET values with annealing for $\mathrm{Cu}$-graphene systems therefore suggests a significant improvement in the interaction between graphene and $\mathrm{Cu}$ and an improvement in the mechanical properties of the interface. There is some controversy in the literature regarding the value of the adhesion strength between graphene and $\mathrm{Cu},{ }^{16-18}$ and on the nature of the interaction leading to this value. There are two main mechanisms that might be used to describe the observed behavior with plasma etching of the graphene-Cu interface: the process of $\mathrm{Cu}$ oxidation itself, or the reconstruction of the $\mathrm{Cu}$ surface with annealing.

The CET as determined for $\mathrm{N}_{2}$ etching seems to be due to the removal of $\mathrm{Cu}$ oxides rather than the graphene layer. Yet the trend of increasing resistance to etch removal with annealing is observed. As $\mathrm{Cu}$ is highly susceptible to oxidation, even at low temperatures, it may be that the oxidation process itself leads to the increased strength of the interaction between $\mathrm{Cu}$ and graphene.

Fundamentally, the oxidation of $\mathrm{Cu}$ can be described by the Cabrera-Mott theory ${ }^{\mathbf{6 0}} \mathrm{Cu}$ ions and electrons migrate to form the oxide layer on the surface, leaving cationic $\mathrm{Cu}$ sites behind. ${ }^{61}$ The strong electric field that results promotes further diffusion of $\mathrm{Cu}$ ions to continue oxidation. ${ }^{61}$

In the composite $\mathrm{Cu}$-graphene system, prolonged annealing may provide more kinetic energy for $\mathrm{Cu}$ ion migration to areas where graphene is absent (Fig. 9). As the vacant $\mathrm{Cu}$ cation sites draw $\mathrm{Cu}$ ions to the oxide-metal interface, they may pile up under the graphene flake. This could result in a stronger coupling of C- $\pi$ orbitals and $\mathrm{Cu}-\mathrm{d}$ orbital hybridization of the graphene- $\mathrm{Cu}$ interface. This bunching of the $\mathrm{Cu}$ under the graphene layers as a result of the oxidation electric field could explain the blue shift observed in the 2D band with annealing (see Fig. 5), attributed to relief of interfacial strain. ${ }^{37,50}$

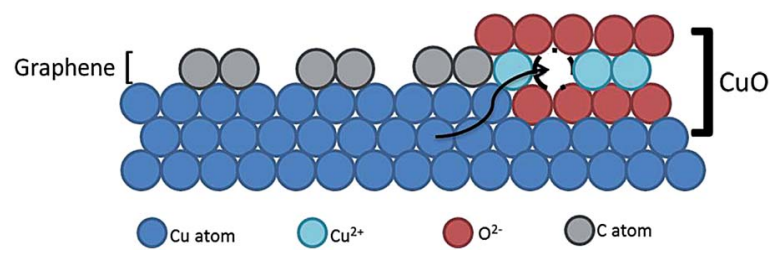

Fig. 9 Schematic of graphene-Cu interface oxidation. 
Another possible mechanism influencing the etch resistance of the postannealed system is the surface reconstruction of the $\mathrm{Cu}$ due to annealing. Rapid contraction of the graphene upon cooling is observed with high temperature $\left(>1000{ }^{\circ} \mathrm{C}\right)$ graphene deposition ${ }^{21,23,24}$ due to the mismatch in the thermal expansion coefficients. ${ }^{25,26}$ This is one of the possible mechanisms for the formation of wrinkles widely observed in graphene-Cu systems. ${ }^{16,21-23}$ Cho et al. and Tian et al. ${ }^{27,28}$ have shown that high temperature post-annealing is able to partially heal the non-equilibrium structure that results from high temperature growth. Zhang et al. ${ }^{21}$ showed that the existence of steps in $\mathrm{Cu}$ under the graphene lowers the adhesion. With annealing induced reconstruction recovering the steps within a $\mathrm{Cu}$ grain $^{27}$ the graphene wrinkles can relax, recovering the Moire reconstruction pattern. ${ }^{22}$ In the equilibrium structure graphene is unwrinkled, and flat graphene flakes are likely to have higher adhesion to the surface compared to wrinkled ones. ${ }^{17,62}$ The observed blue shift, attributed to relief of interfacial strain, ${ }^{50}$ could also result in this case, where the lattice mismatch is changed by the reconstruction of the $\mathrm{Cu}$ surface. Though we used lower temperatures for our post-annealing study, it is possible that a similar, though incomplete, reconstruction occurred in our samples. The increase in interaction strength, as suggested by the robustness to etching, with annealing time therefore could be correlated to the amount of reconstruction.

Further work using friction measurements to clarify these mechanisms is currently under way.

\section{Conclusions}

The graphene grown on $\mathrm{Cu}$ foil by high temperature CVD was annealed after deposition, and exposed to various plasmas. The post-annealing treatment alters the mechanical strength of graphene adhering on the $\mathrm{Cu}$ foil, as shown by the increased resistance to plasma etching. Raman spectroscopy confirms that the graphene is damaged after annealing and plasma treatments, but typically not oxidized during these treatments. The samples were etched and sputtered by air, oxygen, and nitrogen plasmas, yielding different etch rates, appearance and uniformity of the graphene. Nitrogen plasma has a much faster etch rate than air and oxygen due to the mechanism of physical sputtering. For any given plasma however, with post-annealing before plasma exposure, the graphene is harder to remove due to the bonding between the graphene and $\mathrm{Cu}$ substrate. Our results imply that etching of graphene on $\mathrm{Cu}$ can be controlled by proper annealing and the right choice of plasma gas. Plasma etching, with a suitable process gas, appears to be an effective method of estimating the impact of post-processing effects on the mechanical properties of CVD graphene.

\section{Acknowledgements}

The authors acknowledge financial support from 384889-2010 CREAT. The authors are grateful to J. Moran-Mirabal for the Harrick Plasma system, P. Jonasson for the annealing system, S. Kornic for the Raman instrument and CEDT for the microscope support at McMaster University. P. Egberts of U. Calgary is gratefully acknowledged for the insightful discussions. 


\section{Notes and references}

1 C. Lee, X. Wei, J. W. Kysar and J. Hone, Science, 2008, 321, 385-388.

2 Y. S. Dedkov, M. Fonin, U. Rüdiger and C. Laubschat, Appl. Phys. Lett., 2008, 93, 022509.

3 K. S. Novoselov, A. K. Geim, S. V. Morozov, D. Jiang, Y. Zhang, S. V. Dubonos, I. V. Grigorieva and A. A. Firsov, Science, 2004, 306, 666-669.

4 H. Park, R. M. Howden, M. C. Barr, V. Bulović, K. Gleason and J. Kong, ACS Nano, 2012, 6, 6370-6377.

5 Y. Wang, S. W. Tong, X. F. Xu, B. Ozyilmaz and K. P. Loh, Adv. Mater., 2011, 23, 1514-1518.

6 Y. J. Jeong, J. Jang, S. Nam, K. Kim, L. H. Kim, S. Park, T. K. An and C. E. Park, ACS Appl. Mater. Interfaces, 2014, 6, 6816-6824.

7 S. Zhong, J. Q. Zhong, H. Y. Mao, R. Wang, Y. Wang, D. C. Qi, K. P. Loh, A. T. S. Wee, Z. K. Chen and W. Chen, ACS Appl. Mater. Interfaces, 2012, 4, 3134-3140.

8 M. M. Stylianakis, E. Stratakis, E. Koudoumas, E. Kymakis and S. H. Anastasiadis, ACS Appl. Mater. Interfaces, 2012, 4, 4864-4870.

9 Q.-H. Wu, G. Hong, T. W. Ng and S. T. Lee, Appl. Phys. Lett., 2012, 100, 161603.

10 D. Berman, A. Erdemir and A. V. Sumant, Mater. Today, 2014, 17, 31-42.

11 S. Chen, L. Brown, M. Levendorf, W. Cai, S.-Y. Ju, J. Edgeworth, X. Li, C. W. Magnuson, A. Velamakanni, R. D. Piner, J. Kang, J. Park and R. S. Ruoff, ACS Nano, 2011, 5, 1321-1327.

12 E. Sutter, P. Albrecht, F. E. Camino and P. Sutter, Carbon, 2010, 48, 4414-4420.

13 J. S. Bunch, A. M. van der Zande, S. S. Verbridge, I. W. Frank, D. M. Tanenbaum, J. M. Parpia, H. G. Craighead and P. L. McEuen, Science, 2007, 315, 490-493.

14 T. Filleter, J. McChesney, A. Bostwick, E. Rotenberg, K. Emtsev, T. Seyller, K. Horn and R. Bennewitz, Phys. Rev. Lett., 2009, 102, 086102.

15 Y. J. Shin, R. Stromberg, R. Nay, H. Huang, A. T. S. Wee, H. Yang and C. S. Bhatia, Carbon, 2011, 49, 4070-4073.

16 P. Egberts, G. H. Han, X. Z. Liu, A. T. C. Johnson and R. W. Carpick, ACS Nano, 2014, 8, 5010-5021.

17 T. Yoon, W. C. Shin, T. Y. Kim, J. H. Mun, T.-S. Kim and B. J. Cho, Nano Lett., 2012, 12, 1448-1452.

18 X.-Z. Liu, Q. Li, P. Egberts and R. W. Carpick, Adv.Mater. Interfaces, 2014, DOI: 10.1002/admi.201300053.

19 X. Wang, H. You, F. Liu, M. Li, L. Wan, S. Li, Q. Li, Y. Xu, R. Tian, Z. Yu, D. Xiang and J. Cheng, Chem. Vap. Deposition, 2009, 15, 53-56.

20 W. Liu, H. Li, C. Xu, Y. Khatami and K. Banerjee, Carbon, 2011, 49, 4122-4130.

21 Y. Zhang, T. Gao, Y. Gao, S. Xie, Q. Ji, K. Yan, H. Peng and Z. Liu, ACS Nano, 2011, 5, 4014-4022.

22 L. Gao, J. R. Guest and N. P. Guisinger, Nano Lett., 2010, 10, 3512-3516.

23 W. Zhu, T. Low, V. Perebeinos, A. A. Bol, Y. Zhu, H. Yan, J. Tersoff and P. Avouris, Nano Lett., 2012, 12, 3431-3436.

24 L. Zhao, K. T. Rim, H. Zhou, R. He, T. F. Heinz, A. Pinczuk, G. W. Flynn and A. N. Pasupathy, Solid State Commun., 2011, 151, 509-513.

25 D. Yoon, Y. Son and H. Cheong, Nano Lett., 2011, 11, 3227-3231. 
26 F. C. Nix and D. MacNair, Phys. Rev., 1941, 60, 597-605.

27 J. Cho, L. Gao, J. Tian, H. Cao, W. Wu, Q. Yu, E. N. Yitamben, B. Fisher, J. R. Guest, Y. P. Chen and N. P. Guisinger, ACS Nano, 2011, 5, 3607-3613.

28 J. Tian, H. Cao, W. Wu, Q. Yu, N. P. Guisinger and Y. P. Chen, Nano Lett., 2012, 12, 3893-3899.

29 S. D. Costa, A. Righi, C. Fantini, Y. Hao, C. Magnuson, L. Colombo, R. S. Ruoff and M. A. Pimenta, Solid State Commun., 2012, 152, 1317-1320.

30 J. Bai, X. Zhong, S. Jiang, Y. Huang and X. Duan, Nat. Nanotechnol., 2010, 5, 190-194.

31 Y. Wang, Y. Shao, D. W. Matson, J. Li and Y. Lin, ACS Nano, 2010, 4, 1790-1798.

32 M. Wojtaszek, N. Tombros, A. Caretta, P. H. M. van Loosdrecht and B. J. van Wees, J. Appl. Phys., 2011, 110, 063715.

33 D. G. Matei, N.-E. Weber, S. Kurasch, S. Wundrack, M. Woszczyna, M. Grothe, T. Weimann, F. Ahlers, R. Stosch, U. Kaiser and A. Turchanin, Adv. Mater., 2013, 25, 4146-4151.

34 A. Zandiatashbar, G.-H. Lee, S. J. An, S. Lee, N. Mathew, M. Terrones, T. Hayashi, C. R. Picu, J. Hone and N. Koratkar, Nat. Commun., 2014, 5, 3186.

35 G. N. Taylor and T. M. Wolf, Polym. Eng. Sci., 1980, 20, 1087-1092.

36 A. C. Ferrari, J. C. Meyer, V. Scardaci, C. Casiraghi, M. Lazzeri, F. Mauri, S. Piscanec, D. Jiang, K. S. Novoselov, S. Roth and A. K. Geim, Phys. Rev. Lett., 2006, 97, 187401.

37 V. Yu, E. Whiteway, J. Maassen and M. Hilke, Phys. Rev. B: Condens. Matter Mater. Phys., 2011, 84, 205407.

38 J. D. Jones, W. D. Hoffmann, A. V. Jesseph, C. J. Morris, G. F. Verbeck and J. M. Perez, Appl. Phys. Lett., 2010, 97, 233104.

39 I. Pocsik, M. Hundhausen, M. Koos and L. Ley, J. Non-Cryst. Solids, 1998, 10831086.

40 Z. Sun, Z. Yan, J. Yao, E. Beitler, Y. Zhu and J. M. Tour, Nature, 2010, 468, 549552.

41 F. Tuinstra and J. L. Koenig, J. Chem. Phys., 2003, 53, 1126-1130.

42 O. Akhavan, Carbon, 2010, 48, 509-519.

43 J. S. Bunch, S. S. Verbridge, J. S. Alden, A. M. Van Der Zande, J. M. Parpia, H. G. Craighead and P. L. Mceuen, Nano Lett., 2008, 8, 2458-2462.

44 J. Iijima, J.-W. Lim, S.-H. Hong, S. Suzuki, K. Mimura and M. Isshiki, Appl. Surf. Sci., 2006, 253, 2825-2829.

45 A. Ferrari and J. Robertson, Phys. Rev. B: Condens. Matter Mater. Phys., 2000, 61, 14095-14107.

46 F. Zhou, Z. Li, G. J. Shenoy, L. Li and H. Liu, ACS Nano, 2013, 7, 6939-6947.

47 A. Siokou, F. Ravani, S. Karakalos, O. Frank, M. Kalbac and C. Galiotis, Appl. Surf. Sci., 2011, 257, 9785-9790.

48 K. N. Kudin, B. Ozbas, H. C. Schniepp, R. K. Prud'homme, I. A. Aksay and R. Car, Nano Lett., 2008, 8, 36-41.

49 L. M. Malard, M. A. Pimenta, G. Dresselhaus and M. S. Dresselhaus, Phys. Rep., 2009, 473, 51-87.

50 Y. Y. Wang, Z. H. Ni, T. Yu, Z. X. Shen, H. M. Wang, Y. H. Wu, W. Chen and A. T. S. Wee, J. Phys. Chem. C, 2008, 112, 10637-10640.

51 Y. Shao, S. Zhang, M. H. Engelhard, G. Li, G. Shao, Y. Wang, J. Liu, I. A. Aksay and Y. Lin, J. Mater. Chem., 2010, 20, 7491-7496. 
52 M. Lenglet, K. Kartouni, J. Machefert, J. M. Claude, P. Steinmetz, E. Beauprez, J. Heinrich and N. Celati, Mater. Res. Bull., 1995, 30, 393-403.

53 G. Diankov, M. Neumann and D. Goldhaber-Gordon, ACS Nano, 2013, 7, 13241332.

54 V. M. Donnelly and A. Kornblit, J. Vac. Sci. Technol., A, 2013, 31, 050825.

55 J. Herron and D. Green, Plasma Chem. Plasma Process., 2001, 21, 459-481.

56 C. Cardinaud, M. Peignon, P. Tessier and L. Materiaux, Appl. Surf. Sci., 2000, 164, 72-83.

57 D. Manos, D. Flamm, Plasma Etching: An Introduction, 1989.

58 C. Riccardi, R. Barni and M. Fontanesi, J. Appl. Phys., 2001, 90, 3735.

59 A. Hojabri, N. Haghighian, K. Yasserian and M. Ghoranneviss, IOP Conf. Ser.: Mater. Sci. Eng., 2010, 12, 012004.

60 N. Cabrera and N. Mott, Rep. Prog. Phys., 1949.

61 T. N. Rhodin, J. Am. Chem. Soc., 1950, 72, 5102-5106.

62 Z. Ye, C. Tang, Y. Dong and A. Martini, J. Appl. Phys., 2012, 112, 116102. 